Research Article

\title{
The Impact of Age and Pathogens Type on the Gut Microbiota in Infants with Diarrhea in Dalian, China
}

\author{
Qingjie Fan, ${ }^{1}$ Ming Yi, ${ }^{2}$ He Liu, ${ }^{1}$ Yushuang Wang, ${ }^{1}$ Xinke Li, ${ }^{1}$ Jieli Yuan, ${ }^{1}$ Lili Wang, \\ Binbin Hou $\left(\mathbb{0},{ }^{3}\right.$ and Ming $\operatorname{Li}\left(\mathbb{C}^{1}\right.$ \\ ${ }^{1}$ College of Basic Medical Science, Dalian Medical University, Dalian, China \\ ${ }^{2}$ Center for Disease Control (CDC) of Xigang District, Dalian, China \\ ${ }^{3}$ The Second Hospital of Dalian Medical University, Dalian, China \\ Correspondence should be addressed to Binbin Hou; houbinbin1001@163.com and Ming Li; vivianmarat@163.com
}

Received 14 September 2020; Revised 25 October 2020; Accepted 11 November 2020; Published 30 November 2020

Academic Editor: Tingtao Chen

Copyright (c) 2020 Qingiie Fan et al. This is an open access article distributed under the Creative Commons Attribution License, which permits unrestricted use, distribution, and reproduction in any medium, provided the original work is properly cited.

Objective. Diarrhea in infants is a serious gastrointestinal dysfunction characterized by vomiting and watery bowel movements. Without proper treatment, infants will develop a dangerous electrolyte imbalance. Diarrhea is accompanied by intestinal dysbiosis. This study compared the gut microbiota between healthy infants and diarrheic infants. It also investigated the effects of age and pathogen type on the gut microbiota of infants with diarrhea, providing data for the proper treatment for diarrhea in infants. Materials and Methods. DNA was collected from the fecal samples of 42 Chinese infants with diarrhea and 37 healthy infants. The healthy infants and infants with diarrhea were divided into four age groups: 0-120, 120-180, 180-270, and 270-365 days. Using PCR and 16S rRNA high-throughput sequencing, the diarrhea-causing pathogens in these infants were identified and then categorized into four groups: Salmonella infection, Staphylococcus aureus infection, combined Salmonella and Staphylococcus aureus infection, and others (neither Salmonella nor Staphylococcus aureus). Results. The species diversity of gut microbiota in diarrheic infants was significantly reduced compared with that in healthy infants. Infants with diarrhea had a lower abundance of Lactobacillus spp. and Bacillus spp. $(P<0.001)$ and a significant richness of Klebsiella spp. and Enterobacter spp. $(P<0.001)$. Similar gut microbiota patterns were found in diarrheic infants in all four age groups. However, different pathogenic infections have significant effects on the gut microbiota of diarrheic infants. For instance, the relative abundance of Klebsiella spp. and Streptococcus spp. was significantly increased $(P<0.001)$ in infants infected with Staphylococcus aureus; meanwhile, the richness of bacteria such as Enterobacter spp. was significantly increased in the Salmonella infection group $(P<0.001)$. Conclusion. The microbiota in infants with diarrhea has changed significantly, characterized by decreased species diversity and abundance of beneficial bacteria and significant increase in the proportion of conditional pathogens. Meanwhile, the gut microbiota of infants with diarrhea at different ages was similar, but different pathogenic infections affect the gut microbiota characteristics. Therefore, early identification of changes in gut microbiota in infants with diarrhea and the adoption of appropriate pathogen type-specific interventions may effectively alleviate the disease and reduce adverse reactions.

\section{Introduction}

As a serious gastrointestinal dysfunction, infant diarrhea has become a global public health problem. Without proper treatment, the child will have an electrolyte imbalance that can be life-threatening [1]. Diarrhea was the second cause of childhood mortality, according to the last WHO bulletin, published in 2018 (https://www.who.int/gho/publications/ world_health_statistics/2018/en/). Rotavirus, Shigella spp., and Salmonella spp. were the three leading causes of diarrheal deaths in infants [2]. Also, Staphylococcus aureus was one of the common pathogens of infectious diarrhea [3]; the peptidoglycan and toxins of $S$. aureus can induce infantile diarrhea [4].

The gut microbiota community is symbiotic with the host and changes dynamically with the host's age and physiological status as well as environmental factors $[5,6]$. The homeostasis of the intestinal microbiota plays a vital role 
in human health, specifically by promoting the digestion and absorption of food, maintaining the host's immune balance, metabolism, and homeostasis of the intestinal barrier [7, 8]. In addition, the emergence of probiotics, prebiotics, and other products provides new means of preventing and treating clinical diseases $[9,10]$.

However, once the host's intestinal microecological balance was broken, various intestinal diseases will follow [11]. For instance, infantile diarrhea was one of the most common metabolic diseases related to the infant's gut microecological balance. More research has found that gut dysbiosis has an impact on the occurrence and development of diarrhea. For example, The et al. have reported a consistent elevation of Fusobacterium mortiferum, Escherichia, and reduced Bifidobacterium pseudocatenulatum in infants with diarrhea [12]. Another research found Bifidobacterium and Lactobacillus species to be decreased in Colombian children with diarrhea [13]. However, few reports compared the gut microbiota characteristics in infants of different ages or with different pathogens.

This study aimed to (a) identify the differences in the gut microbiota composition between diarrheic (under one year) and healthy infants, (b) characterize the gut microbiota in diarrheic infants of different ages, and (c) examine the effect of different pathogenic bacteria on the intestinal microbiota of infants with diarrhea. This study can clarify gut microbiota changes in infants with diarrhea and provide a specific reference for the additional diagnosis and treatment.

\section{Materials and Methods}

2.1. Subjects and Sample Collection. A total of 42 diarrheic infants and 37 healthy infants under the age of one were recruited from the Center for Disease Control of Xigang District, Dalian, China (Figure 1, Table 1). Infants with virus infection, such as rotavirus, were excluded from the study; all infantile diarrhea cases were caused by prokaryotic infection. Meanwhile, infants with diarrhea were in the early stages of diarrhea and had not been treated with antibiotics. The fecal samples were collected from each infant and immediately stored at $-20^{\circ} \mathrm{C}$ until transfer to the laboratory on dry ice and then stored at $-80^{\circ} \mathrm{C}$ before use.

The study was approved by the ethical committees of Dalian Medical University, Dalian, China. Patients have filled out the informed consent form before sample collection.

\subsection{Fecal DNA Extraction, PCR Amplification, $16 S \mathrm{rRNA}$} Sequencing, and Analysis. The microbial genomic DNA from the fecal samples was extracted using the E.Z.N.A. ${ }^{\circledR}$ Stool DNA kit (Omega Bio-tek, Inc.). The DNA concentration was measured using the Qubit 2.0 Fluorometer (Thermo Fisher Scientific, USA). PCR was performed to amplify the V3 and V4 region of the 16S rRNA gene using the primers $341 \mathrm{~F}\left(5^{\prime}\right.$-CCTAYGGGRBGCASCAG- $\left.3^{\prime}\right)$ and 806R (5'-GGACTACNNGGGTATCTAAT- $\left.3^{\prime}\right)$; template DNA was absent in the negative control [14]. PCR products were monitored on a $2 \%$ agarose gel. The PCR fragments were sequenced on an Illumina HiSeq platform (Novogene, Beijing, China). The QIIME software 1.9 package was used to analyze sequences (Quantitative Insights Into Microbial Ecology, http://bio.cug.edu.cn/qiime/). Sequences having a 97\% resemblance or higher were categorized as the same operational taxonomic units (OTUs). The alpha diversity of microbiota was evaluated by the Chao 1 index, observed species index, and abundance-based coverage estimator (ACE) index. The beta diversity was evaluated by nonmetric multidimensional scaling (NMDS) [15]. The ANOSIM similarity analysis was based on a nonparametric test to compare intragroup and intergroup differences [16]. Linear discriminant analysis Effect Size (LEfSe) was used to identify the bacterial taxa differentially represented between groups at different taxonomic levels. A linear discriminant analysis (LDA) was used to estimate the effect size of each deferentially abundant feature (LDA $\geq 4$ was shown in figures) [17]. The datasets are publicly available (accession number: PRJNA611095).

\subsection{Identification of Different Pathogens in Feces of Diarrheic} Infants. The PCR amplification of the partial 16S rRNA gene of Salmonella and Staphylococcus aureus was performed. The forward (5'-GTG AAA TTA TCG CCA CGT TCG GGC AA- $\left.3^{\prime}\right)$ and reverse primer ( $5^{\prime}$-TCA TCG CA CCG TCA AAG GAA CC- $3^{\prime}$ ) were used to detect a 284-bp Salmonella gene fragment $[18,19]$. Notwithstanding, Staphylococcus aureus infection was identified by the PCR with the forward (5'-AAC TCT GTT ATT AGG GAA GAACA-3') and reverse (5'-CCA CCT TCC GGT TTG TCA CC-3' [20]) primer. This way, we divided the samples into four categories according to pathogen type: Salmonella, Staphylococcus aureus, combined Salmonella and Staphylococcus aureus, and others (neither Salmonella nor Staphylococcus aureus) (Table 1).

2.4. Statistical Analysis. All the experiments were done in triplicate. The data were presented as arithmetic mean\pm standard error of the mean (SEM). Community comparisons were evaluated using a Student's $t$-test with the GraphPad Prism Program (Version 8.1.0; GraphPad Software Inc., La Jolla, CA, USA) [21]. The QIIME was used to calculate the beta diversity distance matrix, and the $R$ language vegan software package was applied to perform NMDS analysis and mapping [22]. A $P$ value of less than 0.05 was considered as statistically significant.

\section{Results}

3.1. Cohorts of Patients and Healthy Infants. The basic clinical information of 37 healthy infants and 42 diarrheic infants was collected, including gender, age, and number of samples in each category. $61.9 \%$ of infants with diarrhea have skin problems, such as pruritus and rash (Table 1). The healthy infants and infants with diarrhea were divided into four groups by age: $0-120,120-180,180-270$, and 270-365 days. Using PCR and 16S rRNA sequencing, the diarrhea-causing pathogens in these infants were identified and divided into 


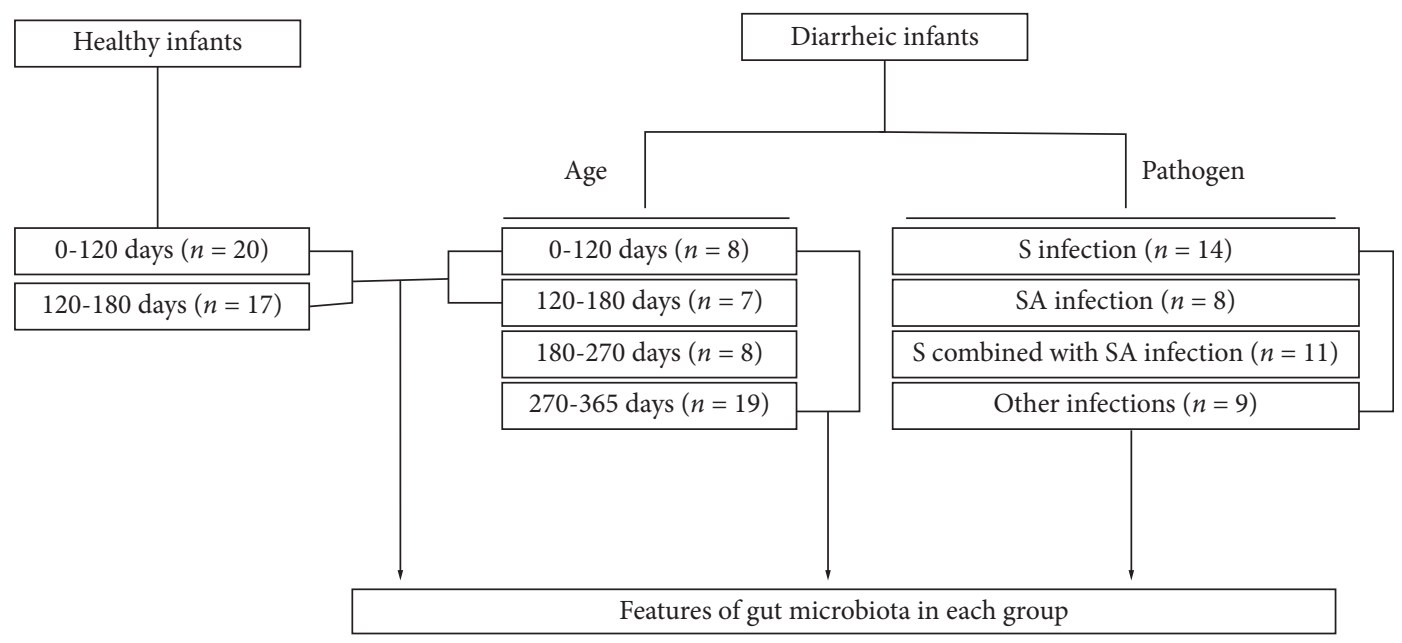

Figure 1: The flowchart of sample collection and grouping in this study. S: Salmonella; SA: Staphylococcus aureus.

TABLE 1: The basic clinical information on healthy infants and infants with diarrhea.

\begin{tabular}{|c|c|c|c|}
\hline \multicolumn{2}{|c|}{ Characteristic } & $\begin{array}{l}\text { Healthy } \\
\text { controls }\end{array}$ & $\begin{array}{l}\text { Diarrheic } \\
\text { infants }\end{array}$ \\
\hline $\begin{array}{l}\text { Number of } \\
\text { samples }\end{array}$ & - & 37 & 42 \\
\hline \multirow{2}{*}{ Gender } & Male & $24(64.9 \%)$ & $22(52.4 \%)$ \\
\hline & Female & $13(35.1 \%)$ & $20(47.6 \%)$ \\
\hline \multirow{4}{*}{ Age } & 0-120 (days) & $20(54.1 \%)$ & $8(19.0 \%)$ \\
\hline & $\begin{array}{c}120-180 \\
\text { (days) }\end{array}$ & $17(45.9 \%)$ & 7 (16.7\%) \\
\hline & $\begin{array}{c}180-270 \\
\text { (days) }\end{array}$ & 0 & $8(19.0 \%)$ \\
\hline & $\begin{array}{c}270-360 \\
\text { (days) }\end{array}$ & 0 & $19(45.2 \%)$ \\
\hline \multirow{4}{*}{ Pathogen } & $S$ & 0 & $14(32.3 \%)$ \\
\hline & SA & 0 & $8(19.0 \%)$ \\
\hline & S.SA & 0 & $11(26.2 \%)$ \\
\hline & Others & 0 & $9(21.4 \%)$ \\
\hline Rash & - & 0 & $26(61.9 \%)$ \\
\hline
\end{tabular}

four categories, Salmonella, Staphylococcus aureus, combined Salmonella and Staphylococcus aureus, and others, which were neither Salmonella nor Staphylococcus aureus (Figure S1). To eliminate the effect of age on experimental results, we excluded 18 diarrheic infants to ensure no statistical difference in the arithmetic mean and SEM deviation of age between diarrheic and the healthy infants $(P=0.897$, Figure 1).

\subsection{The Alterations of Gut Microbiota Composition in Diar-} rheic Infants. The overlapping OTUs of the healthy infant's group and the diarrhea group were shown in a Venn diagram (Figure 2(a)). The $16 \mathrm{~S}$ rRNA gene sequencing showed 977 and 744 unique OTUs in healthy and diarrheic infants, respectively, while 467 OTUs were common in both groups. According to OTU analysis, the bacterial communities in diarrheic and healthy infants tended to be heterogeneous (Figure 2(b)). The ACE, Chao 1, and observed species index showed that gut microbiota of diarrheic infants had significantly lower alpha diversity than those of healthy infants (all $P=0.001$; Figures 2(c), 2(d) and 2(e)).

The NMDS calculation on ranking order was used for clustering the 79 samples into two distinct enterotypes (Figure 3(a)). The intergroup divergence was greater than intragroup divergence (Figure 3(b)), suggesting a significant difference in beta diversity between healthy infants and diarrheic infants. The LDA effect size (LefSe) algorithm was adopted to identify the bacterial groups that showed significant differences in abundance between the two groups. Comparisons between the two groups revealed that the Firmicutes phylum was significantly more abundant in healthy infants than diarrheic infants. At the genus level, the main abundant microbial genera shifted from Lactobacillus and Bifidobacterium in healthy infants to Klebsiella and Streptococcus in diarrheic infants (Figures 3(c) and $3(\mathrm{~d}))$.

Distinct bacterial composition was observed between the healthy and diarrhea group. The microbiome contained 23 phyla, 168 families, and 370 genera in all fecal samples. Proteobacteria, Firmicutes, and Actinobacteria were the most abundant taxonomic groups. The relative abundance of Proteobacteria $(44.67 \%)$ in diarrheic infants was substantially higher than healthy infants $(P<0.001)$, while the abundances of Firmicutes (24.27\%) and Actinobacteria $(22.14 \%)$ in diarrheic infants were lower $(P<0.001$, $P=0.031$, Figures 4(a), and 4(b)). At the family level (Figures 4(c), 4(d)), the relative abundance of Enterobacteriaceae $(43.60 \%)$ in diarrheic infants was considerably higher than in healthy infants $(P<0.001)$. On the contrary, the relative abundance of Lactobacillaceae (3.03\%) and Bifidobacteriaceae $(2.05 \%)$ in diarrheic infants was notably lower than in healthy infants $(P<0.001, P=0.026)$. At the genus level (Figures 4(e), 4(f)), the relative abundance of Klebsiella $(16.57 \%)$ in diarrheic infants was higher than in healthy infants $(P=0.001)$. On the contrary, the relative abundance of Lactobacillus (3.03\%) and Bifidobacterium $(20.52 \%)$ in diarrheic infants was lower than in healthy infants $(P<0.001, P=0.025)$. 


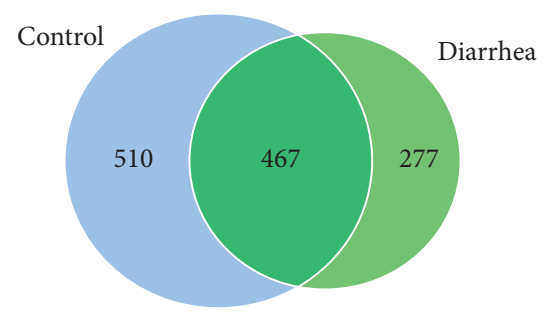

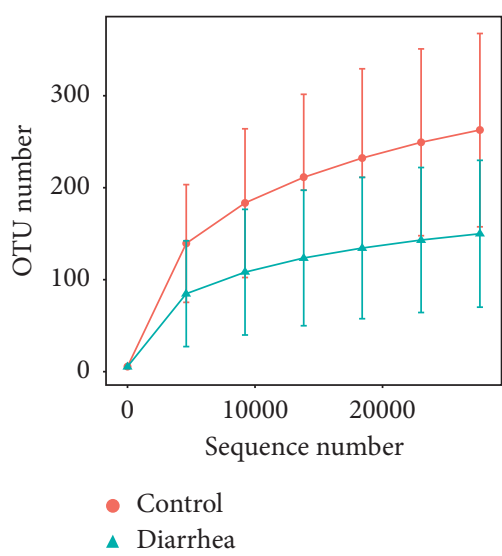

(b)
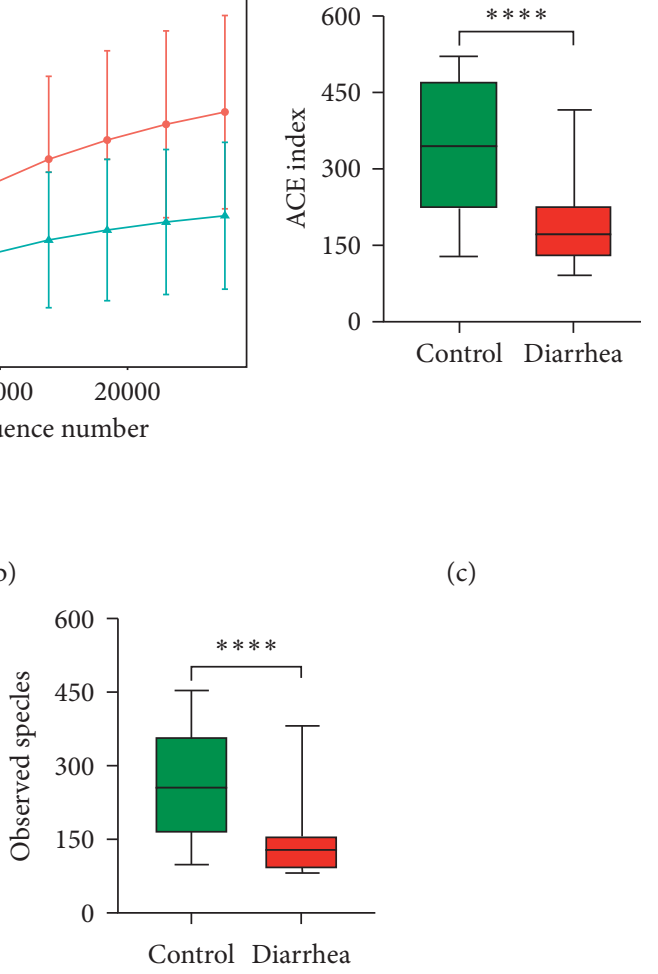

(e)

FIGURE 2: The alpha diversity of gut microbiota in healthy infants and diarrheic infants. (a) Venn diagram of OTUs in the two groups. (b) Observed species index in the two groups. (c-e) Comparing the alpha diversity indices (ACE, Chao 1, and observed species) based on the OTU profiles.

3.3. Characteristics of Gut Microbiota in Healthy and Diarrheic Infants at Different Ages. The healthy infants and infants with diarrhea were divided by age into four groups: 0-120, 120-180, 180-270, and 270-365 days. We investigated the characteristics of the gut microbiota of infants in the four age groups. The alpha diversity indexes showed that, in healthy infants, the diversity of gut microbiota of 120-180-day-old infants was significantly lower than that of $0-120$-day-old infants $(P<0.01)$. Interestingly, different ages did not affect the diversity of the gut microbiota of diarrheic infants (Figure 5(a), $P>0.05$ ). Based on the factor of age, NMDS clustering divided the samples of infants with diarrhea into four groups; however, the four groups did not separate clearly, suggested a similarity among the samples from different age groups (Figure 5(b)).

At the phylum level (Figure 5(c)), the relative abundance of Proteobacteria (54.67\%) in diarrhea-1 (0-120 days) infants was individually higher than in diarrhea-4 (270-365 days) infants $(P=0.027)$. On the contrary, the relative abundance of Actinobacteria (7.29\%) in diarrhea-1 (0-120 days) infants was lower than in diarrhea-3 (180-270 days) infants $(P=0.031)$ and diarrhea-4 (270-365 days) infants $(P=0.013)$. At the family level (Figure $5(\mathrm{~d}))$, the relative abundance of Enterobacteriaceae (53.96\%) in diarrhea-1 infants was significantly higher than in diarrhea- 4 infants $(P=0.025)$. On the contrary, the relative abundance of Bifidobacteriaceae (5.07\%) in diarrhea-1 infants was lower than in diarrhea- 3 infants $(P=0.024)$ and diarrhea- 4 infants $(P=0.009)$. At the genus level (Figure 5(e)), the relative abundance of Bifidobacterium (5.07\%) in diarrhea-1 infants was lower than in diarrhea-3 infants $(P=0.024)$ and diarrhea-4 infants $(P=0.009)$. The relative abundance of Klebsiella (14.42\%) in diarrhea-2 infants (120-180 days) was higher than in diarrhea- 4 infants $(P=0.031)$. Other than the previously mentioned comparisons, the differences among the other groups of diarrheic infants were not statistically significant.

\subsection{The Characteristics of Intestinal Microbiota in Diarrheic} Infants Infected with Different Pathogens. According to Chinese health authorities, Salmonella and Staphylococcus aureus (SA) were the two common causes of infantile diarrhea in China (http://www.phsciencedata.cn/Share/zh$\mathrm{CN} /$ index.jsp). Therefore, we identified the pathogens in the fecal samples of diarrheic infants and divided the samples by pathogen type: Salmonella (S), Staphylococcus aureus (SA), combined Salmonella and Staphylococcus aureus (S.SA), and others (no Salmonella or Staphylococcus aureus). Subsequently, we investigated the characteristics of the intestinal microbiota of diarrheic infants in the four groups. Regardless of the group, the Chao 1 index of diarrheic infants was reduced compared with healthy infants (Figure 6(a)). NMDS clustered the samples of infants with 


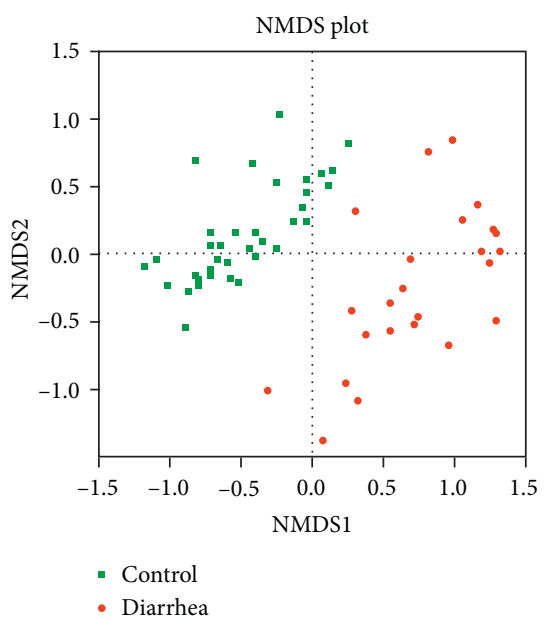

(a)

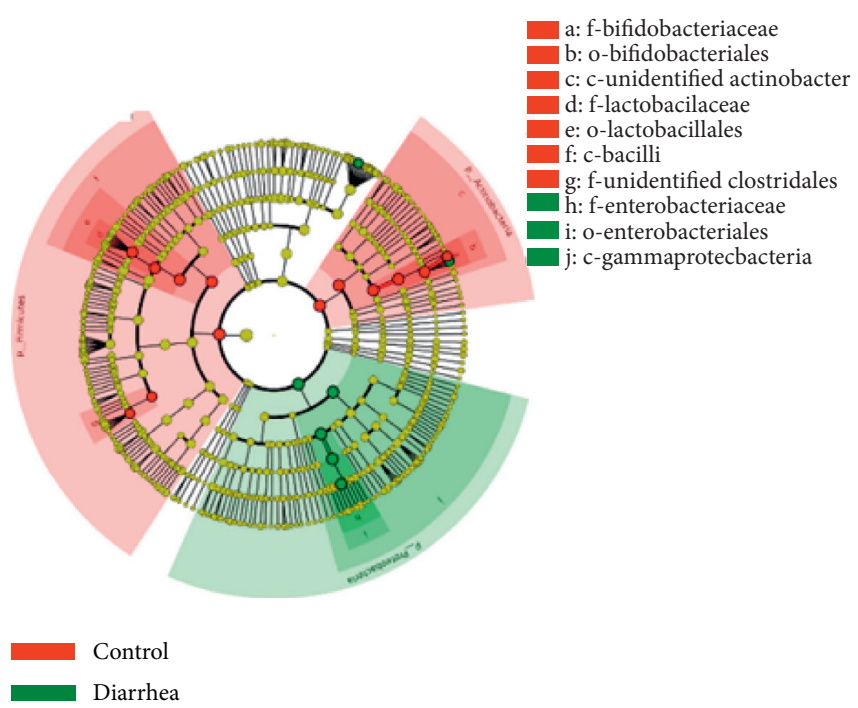

(c)

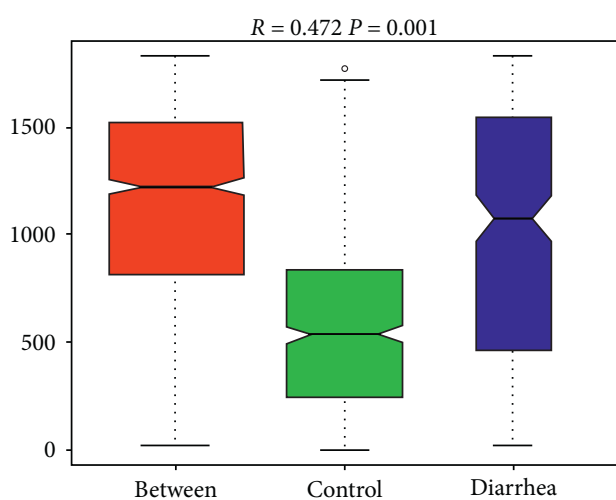

(b)

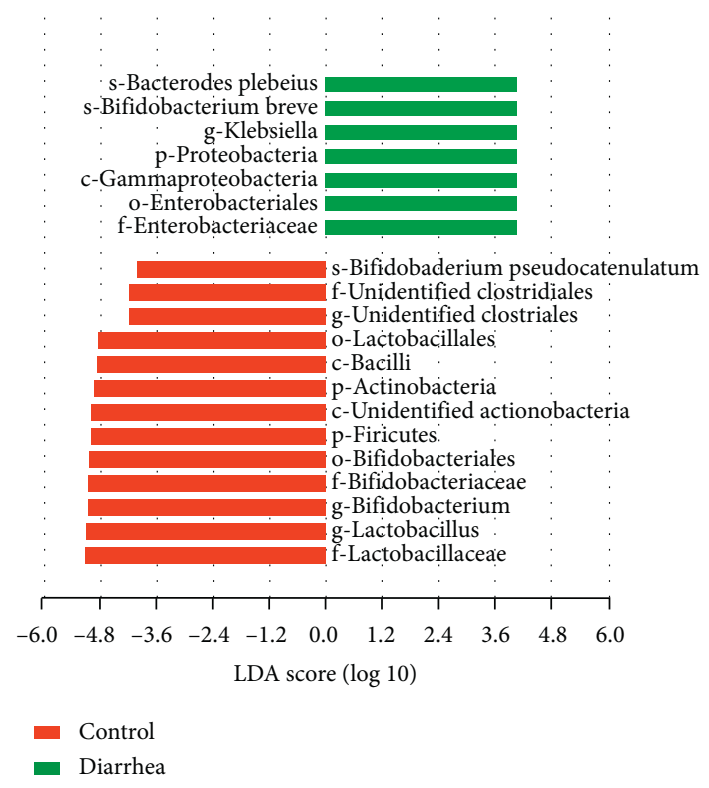

(d)

FIgURE 3: The beta diversity and predominant microbiota of healthy and diarrheic infants. (a) The nonmetric multidimensional scaling (NMDS) of beta diversity is calculated on ranking order. (b) The ANOSIM Similarity Analysis is based on a nonparametric test to compare intragroup and intergroup differences. (c) Cladogram indicating the phylogenetic distribution of microorganisms as related to group characteristics; the difference was shown in red for the healthy group and green for the diarrhea group. The diameter of each circle was proportional to the taxa's abundance. The strategy of multiclass analysis was not strict (at least one somewhat differential). The circle from inside to outside represented the phylogenetic level from domain to genus. (d) Indicator microbial groups within the two types of sediments with a linear discriminate analysis (LDA) value greater than 4.0. The color lump represented the microbes with a significant difference at different taxonomic levels. Red and green represented the healthy group and the diarrhea group, respectively. The $x$-axis represented the LDA score of the microbes. The $y$-axis represented the microbes, which were detected to be significantly different in the groups.

diarrhea into five groups; the Salmonella and Staphylococcus aureus groups were separated for healthy infants but did not separate from each other (Figure 6(b)).

At the genus level, the relative abundance of Lactobacillus and Bifidobacterium in the Salmonella, Staphylococcus aureus, combined Salmonella, and Staphylococcus aureus group was found significantly lower than in healthy infants $(P=0.001)$. In contrast, the Bacteroides and Streptococcus in diarrheic infants were more abundant $(P=0.001)$. Each group had different intestinal microbiota characteristics. For instance, Bifidobacterium and Streptococcus were predominant in the Staphylococcus aureus group, while the Bifidobacterium and Bacteroides account for a large proportion of bacteria in the Staphylococcus aureus group (Figure 6(c)). LEfSe analysis showed that, at the family level, the Ruminococcaceae and Enterobacteriaceae were differentially enriched in the Salmonella group (Figure 6(d)). In addition, at the family level, the most differentially abundant bacteria in healthy infants included Lactobacillaceae and Bifidobacteriaceae, while Streptococcaceae and Ruminococcaceae 

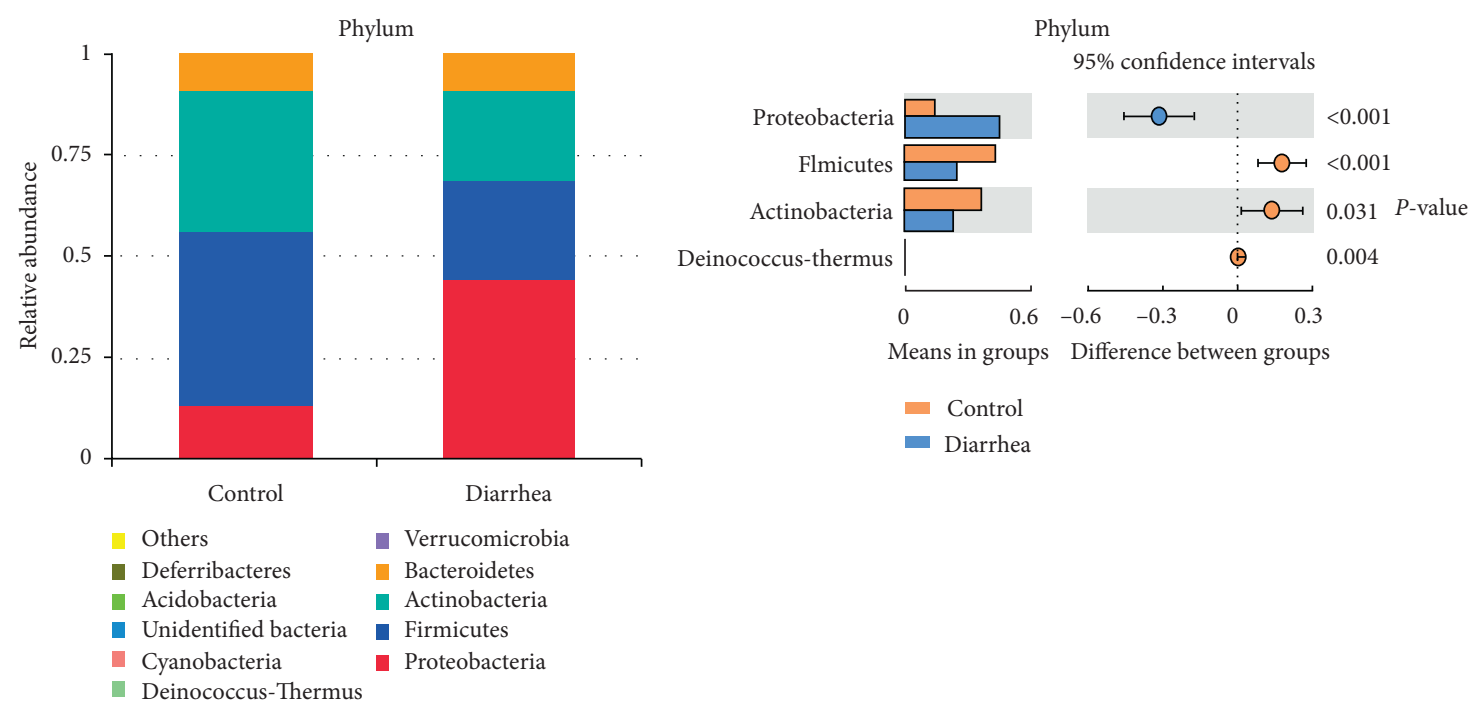

(a)

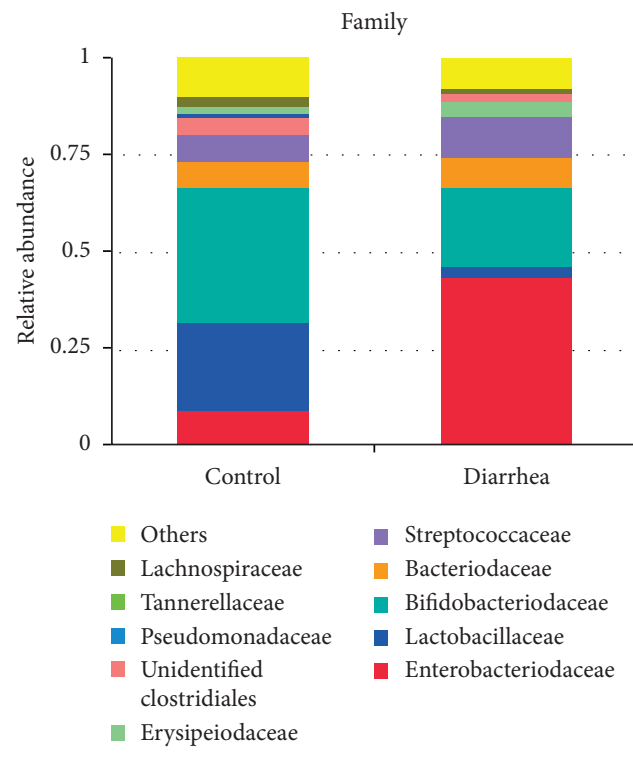

(b)

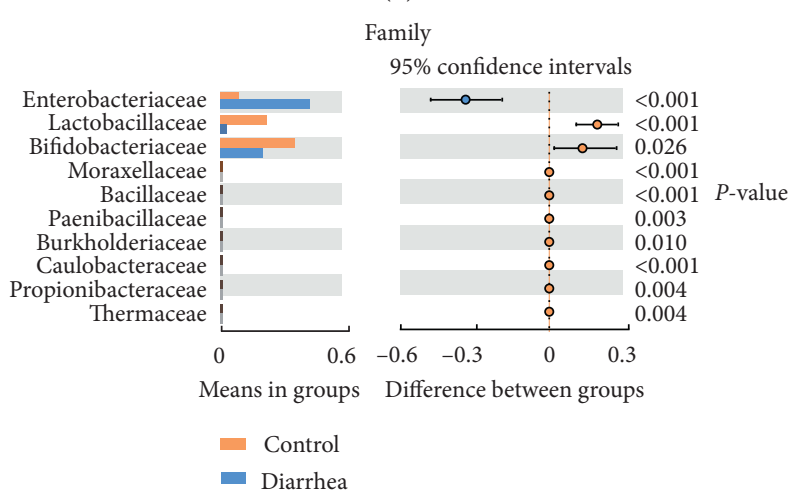

(c)

FIgure 4: Continued. 


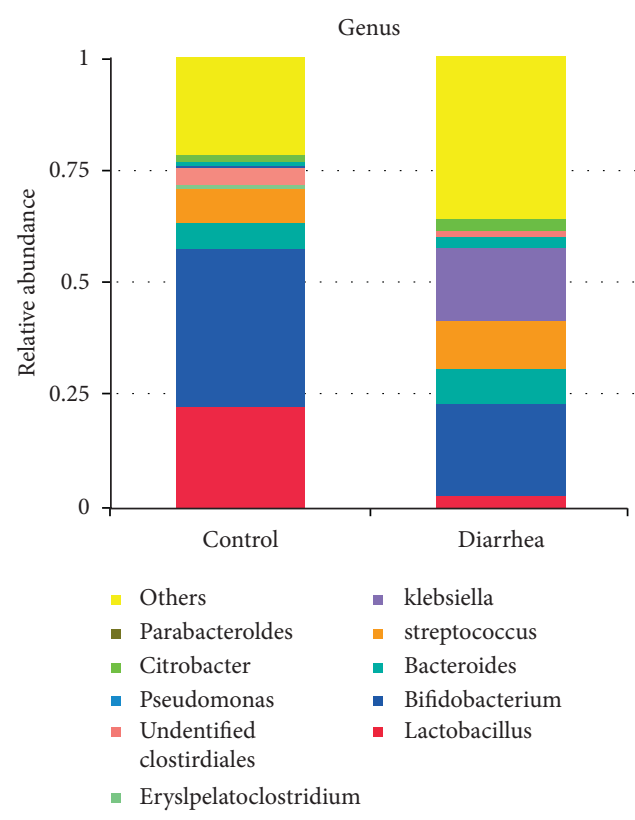

(e)

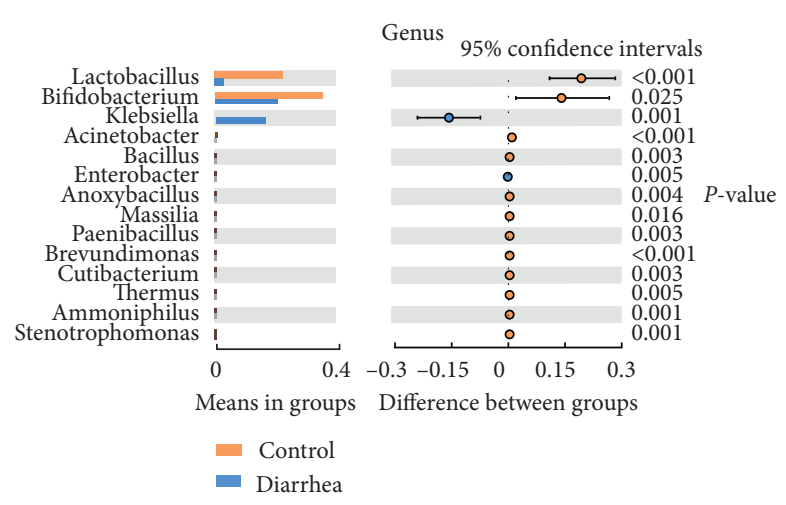

(f)

Figure 4: The shift in the gut microbiota of healthy infants and diarrheic infants. The relative abundance of the top 10 microbiota at the phylum (a), order (c), and genus level (e). The genera in the gut microbiota of healthy infants were strikingly different from those in diarrheic infants at the phylum (b), order (d), and genus level (f).

were overrepresented in the Staphylococcus aureus group (Figure 6(e)). At the genus level, Klebsiella and Streptococcus predominated in the Staphylococcus aureus group, while Faecalibacterium and Subdoligranulum were the predominant bacteria in the Salmonella group (Figure 6(f)).

There were changes in the gut microbiota of the infants infected with different pathogens compared with healthy infants. There were also differences in the gut microbiota between diarrheic infants infected by different pathogens. For instance, at the genus level (Figure 6(c)), the relative abundance of Streptococcus (14.93\%) in the Staphylococcus aureus group was higher than in the Salmonella group $(P=0.017)$ and the other group $(P=0.047)$. Meanwhile, the relative abundance of Enterococcus (1.62\%) in the Staphylococcus aureus group was higher than in the Salmonella group $(P=0.023)$. The relative abundance of Klebsiella (10.45\%) in the combined Salmonella and Staphylococcus aureus group was higher than in the Salmonella group $(P=0.039)$.

\section{Discussion}

The homeostasis of the human gut microbiota has multiple positive effects on the host's health [23]. Microbes colonize the neonatal gut immediately following birth. The establishment and interactive development of the early gut microbiota play a vital role in infants' growth and health [24]. However, during the same period, owing to immune immaturity, the risk of illness will be high $[25,26]$; for example, diarrhea often occurs.

Acute diarrhea was a diarrheal episode of presumed infectious etiology that begins quickly and lasts for fewer than 14 days [27]. Bacterial infections are a common cause of infantile diarrhea. Several different enteropathogenic agents can cause diarrhea in infants, such as enteroaggregative Escherichia coli, enteropathogenic Escherichia coli, Salmonella, Shigella spp., and Staphylococcus aureus, to name a few $[28,29]$.

Our study compared the characteristic of gut microbiota in healthy infants and diarrheic infants in multiple aspects. The results showed that the gut microbiota of infants with diarrhea changed significantly. First, compared with the healthy infants, the intestinal microbiota diversity of diarrheic infants was significantly decreased. Some research revealed that intestinal infection could affect the aerobic bacteria because they would spread through oxygen to obtain energy and metabolism [30-32]. Second, the Firmicutes accounted for a large proportion of bacteria in healthy infants and were beneficial to intestinal epithelial cells $[33,34]$. However, in the diarrhea group, Proteobacteria was the predominant bacteria and the microbial signature of dysbiosis in gut microbiota. Third, our results showed that the relative abundance of Lactobacillus in the healthy infants was significantly higher than that in the diarrheic infants. On the other hand, Enterobacteriaceae had an opposite trend. Recent studies have found that intestinal dysbacteriosis was the leading cause of infantile diarrhea. Lactobacillaceae could alleviate the severity of diarrhea, whereas Enterobacteriaceae had an opposite effect [35]; our results are consistent with this finding.

Finally, when we compare the results of two groups at the genus level, we can find that Lactobacillus decreased and Klebsiella and Enterobacter increased with diarrhea. Lactobacillus are recognized as probiotics because of their health- 

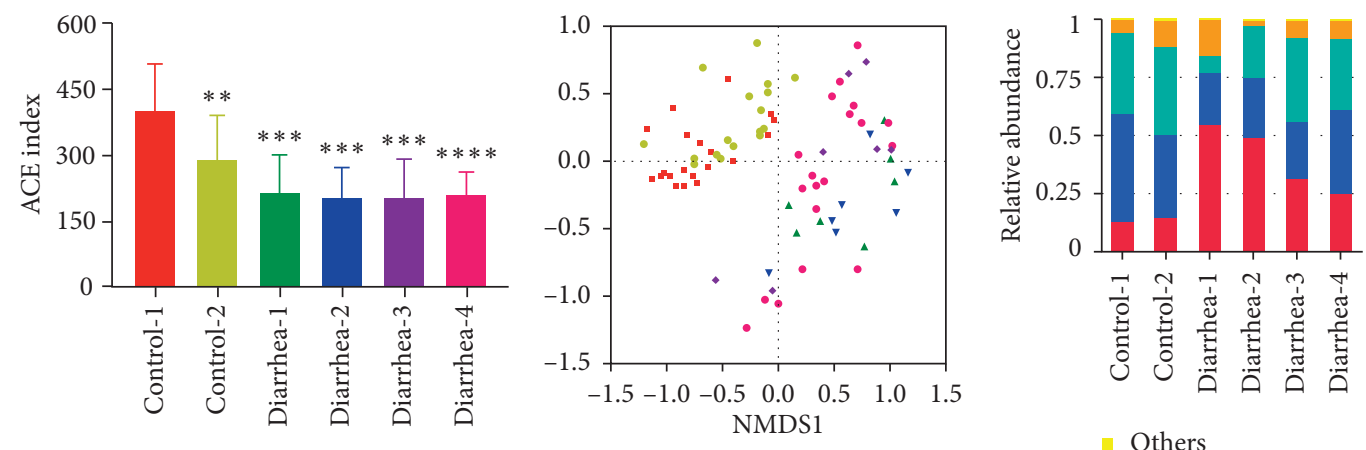

- Control-1 $>$ Diarrhea-2

- Control-2 • Diarrhea-3

^ Diarrhea-1 - Diarrhea-4

In Others

- Acidobacteria

- Fusobacteria

- Unidentified bacteria

- Cyanobacteria

- Deinococcus thermus

- Verrucomicrobia

- Bacteroidetes

- Actinobacteria

- Firmicutes

- Proteobacteria

(a)

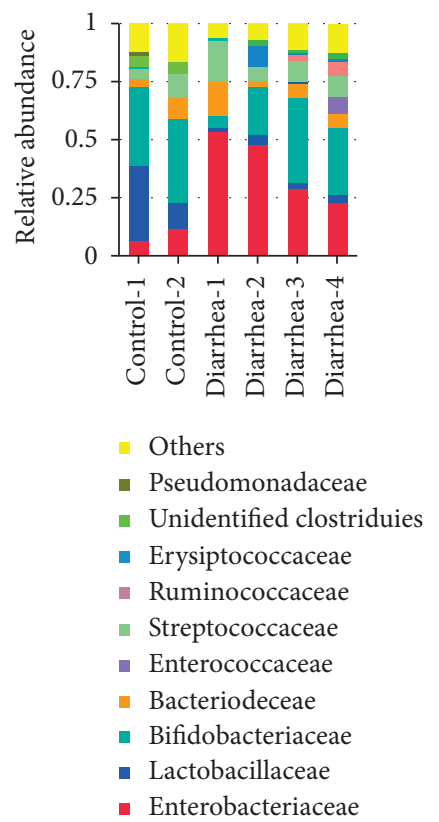

(d) (b)

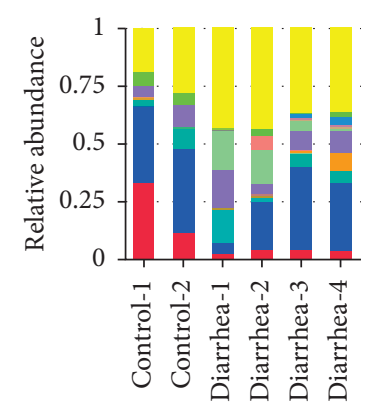

(c)

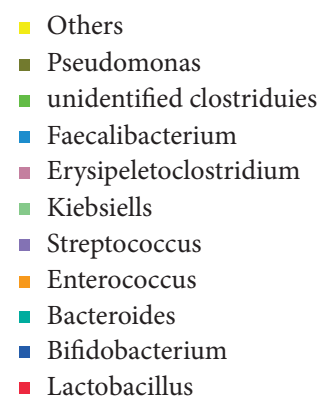

(e)

FIgURE 5: The gut microbiota of healthy infants and infants with diarrhea of different ages. (a). The comparison of the alpha diversity (ACE index) of various ages based on the OTUs profiles. (b). The NMDS of beta diversity is calculated on ranking order. The relative abundance of the top 10 microbiota at the phylum (c), order (d), and genus level (e). Control-1: 0-120-day-old healthy infants; control-2: 120-180-day-old healthy infants; diarrhea-1: 0-120-day-old diarrheic infants; diarrhea-2: 120-180-day-old diarrheic infants; diarrhea-3: 180-270-day-old diarrheic infants; diarrhea-4: 270-360-day-old diarrheic infants.

promoting effects [36]. Lactobacillus can, via competitive exclusion, enhance epithelial barrier function and produce antipathogenic compounds to protect the host [37]. Davoodabadi et al. studied different Lactobacillus strains to identify probiotic candidates for preventing intestinal infections caused by diarrheagenic E. coli [38]. Szajewska et al. found that probiotic Lactobacillus could significantly reduce the risk of antibiotic-associated diarrhea in children and adults $[39,40]$. Numerous bacterial infectious agents have been implicated in $\mathrm{AAD}$, including Clostridium perfringens, Staphylococcus aureus, and Klebsiella oxytoca [41]. $K$. oxytoca also causes infections of the respiratory and urinary tracts and soft-tissue and hepatobiliary infections [42].

We also explored the effects of different ages on the gut microbiota of infants with diarrhea. The results showed no 


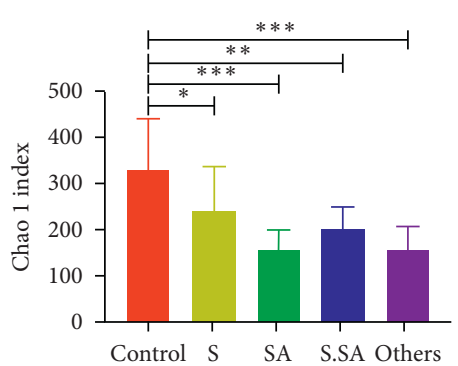

(a)

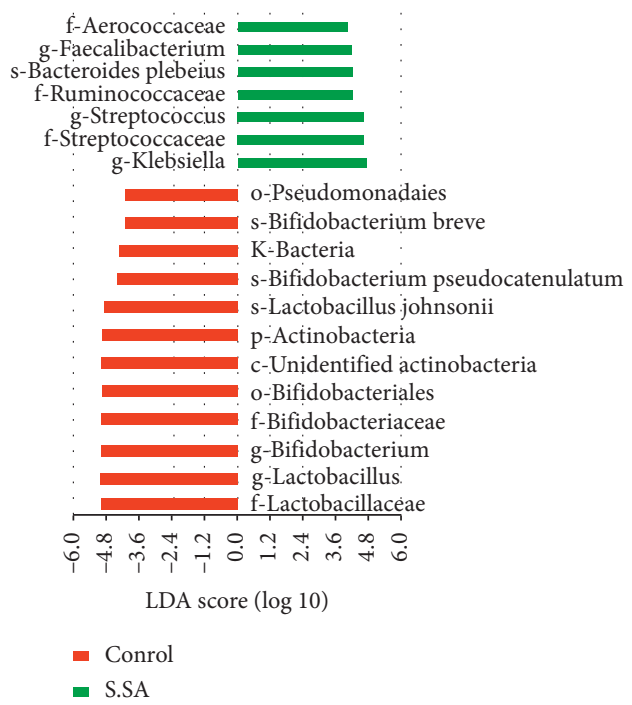

(d)

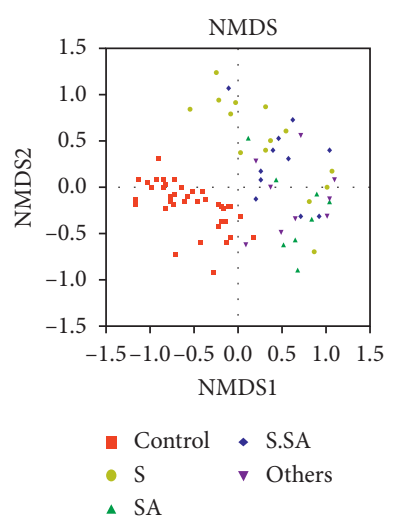

(b)

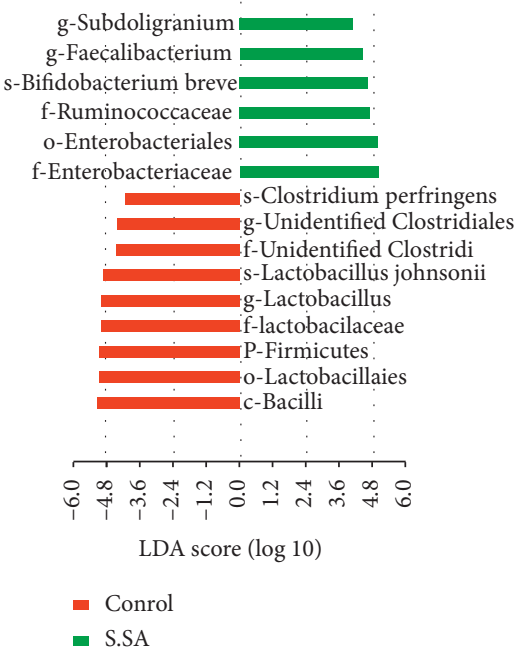

(e)

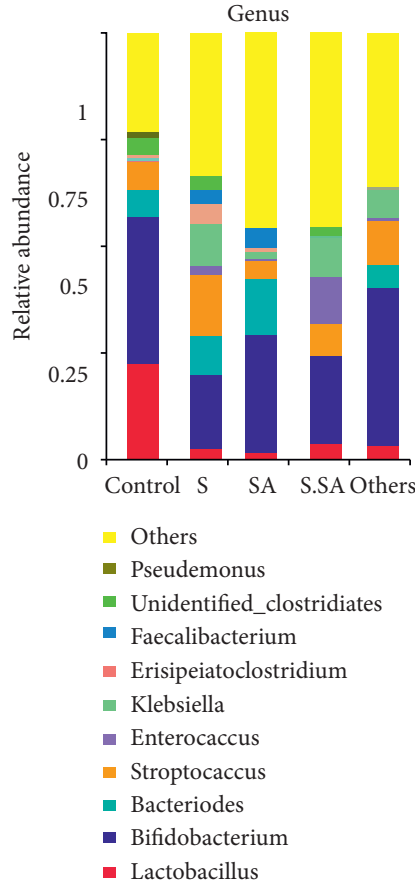

(c)

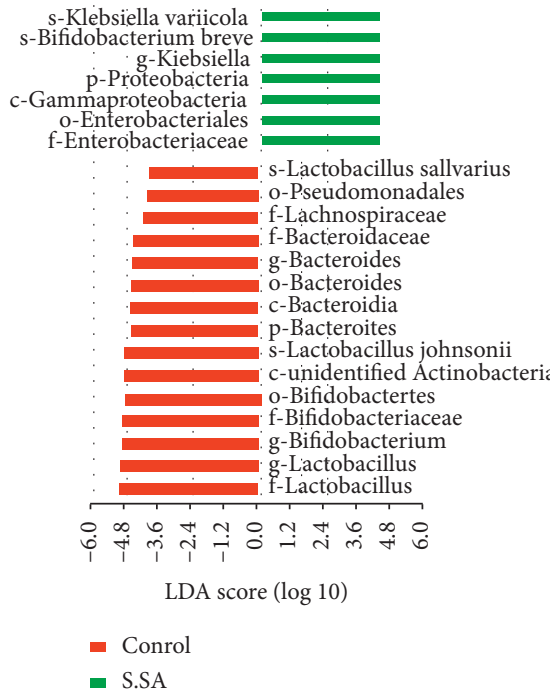

(f)

FIGURE 6: The gut microbiota of diarrheic infants infected by different pathogens. (a). The comparison of the alpha diversity (Chao 1 index) of different groups (control, Salmonella (S), Staphylococcus aureus (SA), combined Salmonella and Staphylococcus aureus (S.SA), and others) based on the OTU profiles. (b). The NMDS of beta diversity was calculated on ranking order. (c). The relative abundance of the top 10 microbiota at the genus level of different groups. (d-f). The LEFSe method analysis of the predominant bacteria between different groups.

significant differences in species diversity among diarrheic infants at different ages. Meanwhile, beta diversity results suggested similar intestinal microbiota in infants with diarrhea at different ages. Interestingly, when we compared the effect of age on the relative abundance of bacterial species, we found differences between the lower and upper age groups. Bifidobacterium's relative abundance was low in the gut microbiota of diarrheic infants of 0-120 days; cesarean delivery may be a factor. Studies have reported that cesarean- section infants show reduced intestinal microbiota complexity and relatively low abundance of Bifidobacterium $[43,44]$.

Meanwhile, the relative abundance of Bifidobacterium increased significantly with age, possibly because the infants had been breastfed since birth. Some studies reported high levels of Bifidobacterium and Lactobacillus in the fecal samples of breastfed infants $[45,46]$. Similarly, we found that, in healthy infants, the relative abundance of 
Lactobacillus was low in the gut microbiota of diarrhea infants from 120 to 180 days, while Bacteroides increased. The reason may be that a newborn's intestine is aerobic, and only facultative anaerobic bacteria can grow. However, in just a few days, the intestinal cavity becomes anaerobic so that only Bifidobacterium, Clostridium, and Bacteroides can colonize [47]. In the first few weeks, the baby's intestinal microbiota resembles the mother's skin and vaginal microbiome, where Enterococci, Streptococcaceae, Lactobacillaceae, Clostridium, and Bifidobacterium predominate. In the first few months, the baby's diet is almost entirely milk, which is conducive to Bifidobacterium, the predominant microbiota at this stage $[48,49]$. When solid food is introduced, the baby's gut microbiota will undergo a substantial change because the food contains various polysaccharides that are not easily digestible; as a result, the abundance of Bacteroides and Clostridium increases and Bifidobacterium and Enterobacteriaceae decrease $[50,51]$. Therefore, it suggested that the changes in the gut microbiota of infants with diarrhea are related to the environment, feeding methods, and delivery methods and are not closely related to age from zero to one year.

Subsequently, based on PCR and sequencing results, we divided diarrheic infants into four groups: Salmonella, Staphylococcus aureus, Salmonella, and Staphylococcus aureus and others. The results of the microbiota analysis showed some differences between the groups. Klebsiella and Staphylococcus were the predominant bacteria in the Staphylococcus aureus group; meanwhile, Enterobacteriaceae was the most abundant bacteria in the Salmonella group. Therefore, diarrhea may be caused by mixed pathogenic bacterial infections. Staphylococcus aureus can secrete staphylococcal enterotoxin A (SEA) [52], which bind to MHC class II molecules and T-cell receptors to stimulate $T$-cell proliferation and activation by the variable region of $\beta$ chain, leading to the uncontrolled increase of many proinflammatory cytokines. The superantigen SEA can cause fever, decrease immunity, and promote many other bacterial infections [53, 54]. Moreover, Staphylococcus aureus's peptidoglycan can promote the occurrence of diarrhea by activating mast cells to release inflammatory substances [55]. Studies have reported reduced Bacteroides and increased Klebsiella in patients with Staphylococcus aureus infection $[56,57]$; such finding is consistent with the results of this study. It is also reported that the use of antibiotics significantly increased the proportion of Klebsiella in intestine [58]. Hence, the cocolonization of Staphylococcus aureus and Klebsiella may be related to antibiotic exposure in the intestinal microenvironment. The relative abundance of Enterobacteriaceae increased, while the relative abundance of Lactobacillus decreased in the Salmonella-infected diarrheic infants; this trend is consistent with the previous finding on the characteristics of intestinal microbiota after Salmonella infection [59-61].

Similarly, patients receiving antibiotics also showed an increase in Enterobacteriaceae [62]. One might wonder why the Salmonella in diarrhea infants promotes Enterobacteriaceae. One possibility is that, after Salmonella infection, the pathogenic factors of Salmonella will be released to induce the host to develop a mucosal inflammation response [63]. As the host tries to eliminate the bacteria, it may cause "collateral damage" that destroys the human intestinal microecological balance, resulting in clinical symptoms like diarrhea.

In this study, we used high-throughput sequencing to investigate gut microbiota's characteristics in infants with diarrhea. We also compared the effects of different ages and different pathogens on the gut microbiota of diarrheic infants. The results showed that the gut microbiota of infants with diarrhea had changed significantly. Simultaneously, different pathogenic infections were found to affect the characteristics of gut microbiota in diarrheic infants; however, the intestinal microbiota of these infants at different ages was similar. This study was our first comprehensive analysis of the effects of different ages and different pathogen types on the gut microbiota in infants with diarrhea. It will provide some reference for the treatment of and nutritional adjustment for diarrheic infants. Indeed, the study's small sample size is a limiting factor; more samples are needed to verify our findings. Also, some infants were treated for skin problems, mostly eczema, followed by urticaria. Since rash in children is closely related to intestinal microbiota disorders and helper $T$ cell imbalance $[64,65]$, the rash's underlying mechanism requires further study.

\section{Data Availability}

All the data that were used to support the findings of this study are included within the article.

\section{Conflicts of Interest}

The authors declare that they have no conflicts of interest.

\section{Authors' Contributions}

M. L. and B. H. conceived the study, designed the experiments, and revised the manuscript. Q. F. performed DNA extraction, analyzed the data, and drafted the manuscript. M.Y. coordinated in selecting field sampling sites and sample collection. H. L., Y. W., and X. L. performed DNA extraction. J. Y. discussed the data. All authors contributed to the critical revision of the manuscript. All authors read and approved the final manuscript.

\section{Acknowledgments}

The authors gratefully acknowledge Professor Li Wenzhe for his technical support. This research was funded by the Nutrition and Care of Maternal and Child Research Fund Project of Guangzhou Biostime Institute of Nutrition and Care (2019BINCMCF02); National Natural Science Foundation of China (no. 31900920); Liaoning Provincial Program for Top Discipline of Basic Medical Sciences, China; Research Project of Liaoning Provincial Department of Education (LZ2020066). 


\section{Supplementary Materials}

Identification of Salmonella infection in infants with diarrhea by agarose gel electrophoresis. PCR product was $284 \mathrm{bp}$. (Supplementary Materials)

\section{References}

[1] J. Gidudu, D. A. Sack, M. Pina et al., "Diarrhea: case definition and guidelines for collection, analysis, and presentation of immunization safety data," Vaccine, vol. 29, no. 5, pp. 1053-1071, 2011.

[2] GBDDD Collaborators, "Estimates of global, regional, and national morbidity, mortality, and aetiologies of diarrhoeal diseases: a systematic analysis for the Global Burden of Disease Study 2015," Lancet Infectious Diseases, vol. 17, no. 9, pp. 909-948, 2017.

[3] G. A. M. Tarr, L. Chui, B. E. Lee et al., "Performance of stooltesting recommendations for acute gastroenteritis when used to identify children with 9 potential bacterial enteropathogens," Clinical Infectious Diseases, vol. 69, no. 7, pp. 1173-1182, 2019.

[4] X. X. Song, D. H. Fang, Y. Q. Quan, and D. J Feng, "The pathogenic detection for 126 children with diarrhea and drug sensitivity tests," European Review for Medical and Pharmacological Sciences, vol. 21, no. 4, pp. 95-99, 2017.

[5] K. Makki, E. C. Deehan, J. Walter, and F. Bäckhed, "The impact of dietary fiber on gut microbiota in host health and disease," Cell Host \& Microbe, vol. 23, no. 6, pp. 705-715, 2018.

[6] G. P. Donaldson, S. M. Lee, and S. K. Mazmanian, "Gut biogeography of the bacterial microbiota," Nature Reviews Microbiology, vol. 14, no. 1, pp. 20-32, 2016.

[7] S. V. Lynch and O. Pedersen, "The human intestinal microbiome in health and disease," New England Journal of Medicine, vol. 375, no. 24, pp. 2369-2379, 2016.

[8] M. Sidhu and D. van der Poorten, "The gut microbiome," Australian Family Physician, vol. 46, no. 4, pp. 206-211, 2017.

[9] H. Okubo, Y. Nakatsu, A. Kushiyama et al., "Gut microbiota as a therapeutic target for metabolic disorders," Current Medicinal Chemistry, vol. 25, no. 9, pp. 984-1001, 2018.

[10] P. Markowiak and K. Śliżewska, "Effects of probiotics, prebiotics, and synbiotics on human health," Nutrients, vol. 9, no. 9, 2017.

[11] A. Gupta, S. Saha, and S. Khanna, "Therapies to modulate gut microbiota: past, present and future," World Journal of Gastroenterology, vol. 26, no. 8, pp. 777-788, 2020.

[12] H. C. The, P. Florez de Sessions, S. Jie et al., "Assessing gut microbiota perturbations during the early phase of infectious diarrhea in Vietnamese children," Gut Microbes, vol. 9, no. 1, pp. 38-54, 2018.

[13] G. Solano-Aguilar, K. P. Fernandez, H. Ets et al., "Characterization of fecal microbiota of children with diarrhea in 2 locations in Colombia," Journal of Pediatric Gastroenterology and Nutrition, vol. 56, no. 5, pp. 503-511, 2013.

[14] A. W. Walker, J. C. Martin, P. Scott et al., "16S rRNA genebased profiling of the human infant gut microbiota is strongly influenced by sample processing and PCR primer choice," Microbiome, vol. 3, p. 26, 2015.

[15] M. C. Hout, M. H. Papesh, and S. D. Goldinger, "Multidimensional scaling," Wiley Interdisciplinary Reviews: Cognitive Science, vol. 4, no. 1, pp. 93-103, 2013.
[16] H. Hermansson, H. Kumar, M. C. Collado et al., "Breast milk microbiota is shaped by mode of delivery and intrapartum antibiotic exposure," Front Nutrition, vol. 6, p. 4, 2019.

[17] N. Segata, J. Izard, L. Waldron et al., "Metagenomic biomarker discovery and explanation," Genome Biology, vol. 12, no. 6, p. R60, 2011.

[18] B. Malorny, J. Hoorfar, C. Bunge, and R. Helmuth, "Multicenter validation of the analytical accuracy of Salmonella PCR: towards an international standard," Applied and Environmental Microbiology, vol. 69, no. 1, pp. 290-296, 2003.

[19] J. Wang, Z. Xu, P. Niu et al., "A two-tube multiplex reverse transcription PCR assay for simultaneous detection of viral and bacterial pathogens of infectious diarrhea," Biomed Research International, vol. 2014, Article ID 648520, 9 pages, 2014.

[20] K. Zhang, J. Sparling, B. L. Chow et al., "New quadriplex PCR assay for detection of methicillin and mupirocin resistance and simultaneous discrimination of Staphylococcus aureus from coagulase-negative staphylococci," Journal of Clinical Microbiology, vol. 42, no. 11, pp. 4947-4955, 2004.

[21] H. J. Motulsky, "Common misconceptions about data analysis and statistics," Pharmacology Research and Perspectives, vol. 3, no. 1, Article ID e00093, 2015.

[22] J. A. Navas-Molina, J. M. Peralta-Sánchez, A. González et al., "Advancing our understanding of the human microbiome using QIIME," Methods in Enzymology, vol. 531, pp. 371-444, 2013.

[23] J. C. Clemente, L. K. Ursell, L. W. Parfrey, and R. Knight, “The impact of the gut microbiota on human health: an integrative view," Cell, vol. 148, no. 6, pp. 1258-1270, 2012.

[24] S. Rautava, R. Luoto, S. Salminen, and E. Isolauri, "Microbial contact during pregnancy, intestinal colonization and human disease," Nature Reviews Gastroenterology \& Hepatology, vol. 9, no. 10, pp. 565-576, 2012.

[25] T. Itani, C. Ayoub Moubareck, I. Melki et al., "Establishment and development of the intestinal microbiota of preterm infants in a Lebanese tertiary hospital," Anaerobe, vol. 43, pp. 4-14, 2017.

[26] S. Arboleya, B. Sánchez, C. Milani et al., "Intestinal microbiota development in preterm neonates and effect of perinatal antibiotics," The Journal of Pediatrics, vol. 166, no. 3, pp. 538-544, 2015.

[27] Bull World Health Organ, "Persistent diarrhoea in children in developing countries: memorandum from a WHO meeting," Bull World Health Organ, vol. 66, no. 6, pp. 709-717, 1988.

[28] D. Law, "Adhesion and its role in the virulence of enteropathogenic Escherichia coli," Clinical Microbiology Reviews, vol. 7, no. 2, pp. 152-173, 1994.

[29] P. C. Okhuysen and H. L. Dupont, "EnteroaggregativeEscherichia coli(EAEC): a cause of acute and persistent diarrhea of worldwide importance," The Journal of Infectious Diseases, vol. 202, no. 4, pp. 503-505, 2010.

[30] T. Conway and P. S. Cohen, "Commensal and pathogenic escherichia coli metabolism in the Gut," Microbiology Spectrum, vol. 3, no. 3, 2015.

[31] R. Gao, C. Zhu, H. Li et al., "Dysbiosis signatures of gut microbiota along the sequence from healthy, young patients to those with overweight and obesity," Obesity, vol. 26, no. 2, pp. 351-361, 2018.

[32] A. Swidsinski, V. Loening-Baucke, H. Verstraelen, S. Osowska, and Y. Doerffel, "Biostructure of fecal microbiota in healthy subjects and patients with chronic idiopathic diarrhea," Gastroenterology, vol. 135, no. 2, pp. 568-579, 2008.

[33] J.-P. Segain, D. Raingeard de la Bletiere, A. Bourreille et al., "Butyrate inhibits inflammatory responses through NFkappa 
B inhibition: implications for Crohn's disease," Gut, vol. 47, no. 3, pp. 397-403, 2000.

[34] P. Louis and H. J. Flint, "Diversity, metabolism and microbial ecology of butyrate-producing bacteria from the human large intestine," FEMS Microbiology Letters, vol. 294, no. 1, pp. 1-8, 2009.

[35] X. Yin, X. Gu, T. Yin, H. Wen, X. Gao, and X. Zheng, "Study of enteropathogenic bacteria in children with acute diarrhoea aged from 7 to 10 years in Xuzhou, China," Microbial Pathogenesis, vol. 91, pp. 41-45, 2016.

[36] Z. Zhang, J. Lv, L. Pan, and Y. Zhang, "Roles and applications of probiotic Lactobacillus strains," Applied Microbiology and Biotechnology, vol. 102, no. 19, pp. 8135-8143, 2018.

[37] B. Sanchez, S. Delgado, A. Blanco-Miguez et al., "Probiotics, gut microbiota, and their influence on host health and disease," Molecular Nutrition \& Food Research, vol. 61, no. 1, 2017.

[38] A. Davoodabadi, M. M. Soltan Dallal, A. Rahimi Foroushani, M. Douraghi, M. k. Sharifi Yazdi, and F. Amin Harati, "Antibacterial activity of Lactobacillus spp. isolated from the feces of healthy infants against enteropathogenic bacteria," Anaerobe, vol. 34, pp. 53-58, 2015.

[39] H. Szajewska and M. Kołodziej, "Systematic review with metaanalysis:Saccharomyces boulardiiin the prevention of antibiotic-associated diarrhoea," Alimentary Pharmacology \& Therapeutics, vol. 42, no. 7, pp. 793-801, 2015.

[40] H. Szajewska and M. Kołodziej, "Systematic review with metaanalysis: Lactobacillus rhamnosusGG in the prevention of antibiotic-associated diarrhoea in children and adults," Alimentary Pharmacology \& Therapeutics, vol. 42, no. 10, pp. 1149-1157, 2015.

[41] K. Flemming and G. Ackermann, "Prevalence of enterotoxin producing Staphylococcus aureus in stools of patients with nosocomial diarrhea," Infection, vol. 35, no. 5, pp. 356-358, 2007.

[42] G. Gorkiewicz, "Nosocomial and antibiotic-associated diarrhoea caused by organisms other than Clostridium difficile," International Journal of Antimicrobial Agents, vol. 33, no. 1, pp. S37-S41, 2009.

[43] M.-J. Butel, A.-J. Waligora-Dupriet, and S. Wydau-Dematteis, "The developing gut microbiota and its consequences for health," Journal of Developmental Origins of Health and Disease, vol. 9, no. 6, pp. 590-597, 2018.

[44] Y. Shao, S. C. Forster, E. Tsaliki et al., "Stunted microbiota and opportunistic pathogen colonization in caesarean-section birth," Nature, vol. 574, no. 7776, pp. 117-121, 2019.

[45] S. A. Lee, J. Y. Lim, B.-S. Kim et al., "Comparison of the gut microbiota profile in breast-fed and formula-fed Korean infants using pyrosequencing," Nutrition Research and Practice, vol. 9, no. 3, pp. 242-248, 2015.

[46] S. Amarri, F. Benatti, M. L. Callegari et al., "Changes of gut microbiota and immune markers during the complementary feeding period in healthy breast-fed infants," Journal of $\mathrm{Pe}$ diatric Gastroenterology and Nutrition, vol. 42, no. 5, pp. 488-495, 2006.

[47] S. Matamoros, C. Gras-Leguen, F. Le Vacon, G. Potel, and M.-F. de La Cochetiere, "Development of intestinal microbiota in infants and its impact on health," Trends in Microbiology, vol. 21, no. 4, pp. 167-173, 2013.

[48] S. J. Lahtinen, R. J. Boyle, S. Kivivuori et al., "Prenatal probiotic administration can influence Bifidobacterium microbiota development in infants at high risk of allergy," Journal of Allergy and Clinical Immunology, vol. 123, no. 2, pp. 499-501, 2009.
[49] F. Turroni, C. Peano, D. A. Pass et al., "Diversity of bifidobacteria within the infant gut microbiota," PLoS One, vol. 7, no. 5, Article ID e36957, 2012.

[50] J. E. Koenig, A. Spor, N. Scalfone et al., "Succession of microbial consortia in the developing infant gut microbiome," Proceedings of the National Academy of Sciences, vol. 108, no. 1, pp. 4578-4585, 2011.

[51] M. Fallani, S. Amarri, A. Uusijarvi et al., "Determinants of the human infant intestinal microbiota after the introduction of first complementary foods in infant samples from five European centres," Microbiology, vol. 157, no. 5, pp. 1385-1392, 2011.

[52] Y. Le Loir, F. Baron, and M. Gautier, "Staphylococcus aureus and food poisoning," The Genetics and Molecular Research Journal, vol. 2, no. 1, pp. 63-76, 2003.

[53] M. Á. Argudín, M. C. Mendoza, and M. R. Rodicio, "Food poisoning and Staphylococcus aureus enterotoxins," Toxins, vol. 2, no. 7, pp. 1751-1773, 2010.

[54] E. Larkin, R. Carman, T. Krakauer, and B. Stiles, "Staphylococcus aureus: the toxic presence of a pathogen extraordinaire," Current Medicinal Chemistry, vol. 16, no. 30, pp. 4003-4019, 2009.

[55] B.-S. Feng, S.-H. He, P.-Y. Zheng, L. Wu, and P.-C. Yang, "Mast cells play a crucial role in Staphylococcus aureus peptidoglycan-induced diarrhea," The American Journal of Pathology, vol. 171, no. 2, pp. 537-547, 2007.

[56] D. Dong, Q. Ni, C. Wang et al., "Effects of intestinal colonization by Clostridium difficile and Staphylococcus aureus on microbiota diversity in healthy individuals in China," $B M C$ Infectious Diseases, vol. 18, no. 1, p. 207, 2018.

[57] J. A. Garfias-López, G. Castro-Escarpuli, P. E. Cárdenas, M. M. B. Moreno-Altamirano, J. Padierna-Olivos, and F. J. Sánchez-García, "Immunization with intestinal microbiota-derived Staphylococcus aureus and Escherichia coli reduces bacteria-specific recolonization of the intestinal tract," Immunology Letters, vol. 196, pp. 149-154, 2018.

[58] A. Rehman, F.-A. Heinsen, M. E. Koenen et al., "Effects of probiotics and antibiotics on the intestinal homeostasis in a computer controlled model of the large intestine," $B M C$ Microbiology, vol. 12, no. 1, p. 47, 2012.

[59] M. Barman, D. Unold, K. Shifley et al., "Enteric salmonellosis disrupts the microbial ecology of the murine gastrointestinal tract," Infection and Immunity, vol. 76, no. 3, pp. 907-915, 2008.

[60] B. L. Deatherage Kaiser, J. Li, J. A. Sanford et al., "A multiomic view of host-pathogen-commensal interplay in salmonella-mediated intestinal infection," PLoS One, vol. 8, no. 6, Article ID e67155, 2013.

[61] B. Stecher, S. Chaffron, R. Kappeli et al., "Like will to like: abundances of closely related species can predict susceptibility to intestinal colonization by pathogenic and commensal bacteria," PLoS Pathog, vol. 6, no. 1, Article ID e1000711, 2010.

[62] C. Ubeda, Y. Taur, R. R. Jenq et al., "Vancomycin-resistant Enterococcus domination of intestinal microbiota is enabled by antibiotic treatment in mice and precedes bloodstream invasion in humans," Journal of Clinical Investigation, vol. 120, no. 12, pp. 4332-4341, 2010.

[63] J. Fritsch and M. T. Abreu, "The microbiota and the immune response: what is the chicken and what is the egg?" Gastrointestinal Endoscopy Clinics of North America, vol. 29, no. 3, pp. 381-393, 2019.

[64] A. K. Leung, K. L. Hon, K. F. Leong, and C. M Sergi, "Measles: a disease often forgotten but not gone," Hong Kong Medical 
Journal = Xianggang Yi Xue Za Zhi, vol. 24, no. 5, pp. 512-520, 2018.

[65] P. V. Kirjavainen, S. J. Salminen, and E. Isolauri, "Probiotic bacteria in the management of atopic disease: underscoring the importance of viability," Journal of Pediatric Gastroenterology and Nutrition, vol. 36, no. 2, pp. 223-227, 2003. 